\title{
FGFR3 Rearrangement Analysis
}

National Cancer Institute

\section{Source}

National Cancer Institute. FGFR3 Rearrangement Analysis. NCI Thesaurus. Code C157193.

A procedure used to detect and identify rearrang ements involving the FGFR3 gene. 